\title{
ON INDECOMPOSABLE DECOMPOSITIONS OF CS-MODULES
}

\author{
NGUYEN V. DUNG
}

(Received 26 April 1994; revised 14 November 1994)

Communicated by P. Schultz

\begin{abstract}
It is shown that, over any ring $R$, the direct sum $M=\oplus_{i \in I} M_{i}$ of uniform right $R$-modules $M_{i}$ with local endomorphism rings is a CS-module if and only if every uniform submodule of $M$ is essential in a direct summand of $M$ and there does not exist an infinite sequence of non-isomorphic monomorphisms $M_{i_{1}} \stackrel{f_{1}}{\rightarrow} M_{i_{2}} \stackrel{f_{2}}{\rightarrow} \cdots \rightarrow M_{i_{n}} \stackrel{f_{n}}{\rightarrow} \cdots$, with distinct $i_{n} \in I$. As a consequence, any CS-module which is a direct sum of submodules with local endomorphism rings has the exchange property.
\end{abstract}

1991 Mathematics subject classification (Amer. Math. Soc.): primary 16D70; secondary 16D50, 16L60.

\section{Introduction}

A module $M$ is called a CS-module (or extending module) if every submodule of $M$ is essential in a direct summand of $M$. CS-modules have been studied extensively in recent years (see for example $[2,3,5,7,8,12,14,15,17,18]$ ), and it appears that several classical theorems on injective modules have natural generalizations for CS-modules. However, in some sense, the CS property is quite far from injectivity and several questions on CS-modules still remain unsolved. An interesting open question is to find necessary and sufficient conditions for a CS-module to have an indecomposable decomposition (see [14, Open problem 8, p. 106]). A very closely related question is to determine when a direct sum of indecomposable modules is a CS-module. The purpose of this paper is to settle this latter question in the case when each indecomposable summand has a local endomorphism ring.

More precisely, we prove that, over any ring $R$, the direct sum $M=\oplus_{i \in I} M_{i}$ of uniform right $R$-modules $M_{i}$ with local endomorphism rings is a CS-module if and only if every uniform submodule of $M$ is essential in a direct summand of $M$ and there does not exist an infinite sequence $M_{i_{1}} \stackrel{f_{1}}{\rightarrow} M_{i_{2}} \stackrel{f_{2}}{\rightarrow} \cdots \rightarrow M_{i_{n}} \stackrel{f_{n}}{\rightarrow} \cdots$ of non- 
isomorphic monomorphisms $f_{n}$, with distinct $i_{n} \in I$. As a consequence, we deduce that if $M$ is a CS-module which has an indecomposable decomposition $M=\bigoplus_{i \in I} M_{i}$, where each $M_{i}$ has a local endomorphism ring, then this decomposition complements direct summands and $M$ has the exchange property. A special case of this corollary was essentially observed earlier in Kamal and Müller [12, Theorem 12], where the ring was assumed to be right Noetherian.

There is another motivation for the paper. A well-known open problem asks whether a (left and right) perfect right self-injective ring $R$ must be quasi-Frobenius, or briefly QF (if $R$ is also left self-injective, the answer is "yes" by Kato [13] and Osofsky [16]). To prove that such $R$ is $\mathrm{QF}$ is equivalent to showing that each projective right $R$-module is injective, that is any infinite direct sums of indecomposable injective projective right $R$-modules are also injective. This was the approach in Clark and Huynh [4] where it was proved that a semiperfect right self-injective ring $R$ is QF if and only if every uniform submodule of any projective right $R$-module is contained in a finitely generated submodule. We will show that this characterization of QF-rings can be deduced as an immediate consequence of our results on CS-modules, but our method of proof is quite different.

\section{Definitions and preliminaries}

Throughout this paper, all rings are associative with identity and all modules are unitary right modules.

A submodule $N$ of a module $M$ is said to be essential in $M$ if $N \cap K \neq 0$ for every nonzero submodule $K$ of $M$. A nonzero module $M$ is called uniform if every nonzero submodule of $M$ is essential in $M$. A submodule $C$ of $M$ is called a complement submodule in $M$ provided $C$ has no proper essential extensions in $M$.

Following [3], a module $M$ is called a CS-module if every complement submodule of $M$ is a direct summand of $M$, or equivalently, every submodule of $M$ is essential in a direct summand of $M$. It is obvious that an indecomposable module is CS if and only if it is uniform.

A non-empty family $\left\{A_{i} \mid i \in I\right\}$ of submodules of a module $M$ is called a local direct summand of $M$ if $\sum_{i \in I} A_{i}$ is direct and $\sum_{i \in F} A_{i}$ is a direct summand of $M$ for any finite subset $F \subseteq I$. If, furthermore, $\sum_{i \in I} A_{i}$ is a direct summand of $M$, then we say that the local direct summand $\left\{A_{i} \mid i \in I\right\}$ is a direct summand of $M$. A family of modules $\left\{M_{i} \mid i \in I\right\}$ is called locally semi-T-nilpotent if, for any countable set of non-isomorphisms $\left\{f_{n}: M_{i_{n}} \rightarrow M_{i_{n+1}}\right\}$ with all $i_{n}$ distinct in $I$, and for any $x \in M_{i_{1}}$, there exists $k$ (depending on $x$ ) such that $f_{k} \cdots f_{1}(x)=0$.

A module $M$ is said to have the exchange property (Crawley and Jónsson [6]) if for any index set $I$, whenever $M \oplus N=\oplus_{i \in I} A_{i}$ for modules $N$ and $A_{i}$, then 
$M \oplus N=M \oplus\left(\oplus_{i \in l} B_{i}\right)$ for submodules $B_{i} \subseteq A_{i}$. A decomposition $M=\oplus_{i \in I} M_{i}$ is said to complement direct summands (Anderson and Fuller [1]) if, for any direct summand $A$ of $M$, there exists a subset $J \subseteq I$ such that $M=A \oplus\left(\oplus_{i \in J} M_{i}\right)$.

For the reader's convenience, we list now a number of known results which will be used in the next section.

LEMMA 2.1. An indecomposable module $M$ has the exchange property if and only if End $M$ is local.

Proof. See Warfield [19, Proposition 1].

LEMMA 2.2. Let $M=\oplus_{i \in I} M_{i}$ be a direct sum of modules with local endomorphism rings. Then the following are equivalent:

(a) $S=$ End $M$ is a semi-regular ring, that is $S / J(S)$ is von Neumann regular and the idempotents in $S / J(S)$ can be lifted over $J(S)$, where $J(S)$ is the Jacobson radical of $S$;

(b) Every local direct summand of $M$ is a direct summand;

(c) The decomposition $M=\oplus_{i \in I} M_{i}$ complements direct summands;

(d) The family $\left\{M_{i} \mid i \in I\right\}$ is locally semi-T-nilpotent;

(e) $M$ has the exchange property;

PROOF. The equivalence of (a), (b), (c) and (d) is due to Harada [11]. The equivalence of (d) and (e) is due to Zimmermann-Huisgen and Zimmermann [22].

Let $R$ be a ring and $M$ a right $R$-module. For each subset $X$ of $M$, we denote the annihilator of $X$ in $R$ by

$$
r_{R}(X)=\{r \in R \mid x r=0 \text { for all } x \in X\} .
$$

When there is no ambiguity, we write $r(X)$ instead of $r_{R}(X)$.

The chain condition in the next lemma appeared as the condition $\left(A_{2}\right)$ in Mohamed and Müller [14, p.4].

LEMMA 2.3. Let $\left\{M_{i} \mid i \in I\right\}$ be right $R$-modules. Then $\oplus_{i \in I} M_{i}$ is quasi-injective if and only if $M_{i}$ is $M_{j}$-injective for all $i, j \in I$ and for every choice of $x_{n} \in M_{i_{n}}$, with distinct $i_{n} \in I$, such that $\cap_{n=1}^{\infty} r_{R}\left(x_{n}\right) \supseteq r_{R}(y)$ for some $y \in M_{j}(j \in I)$, the ascending sequence $\cap_{k=n}^{\infty} r_{R}\left(x_{k}\right)(n \in \mathbb{N})$ becomes stationary.

ProOF. See Mohamed and Müller [14, Proposition 1.18] 


\section{CS-modules which are direct sums of modules with local endomorphism rings}

Our primary aim in this section is to establish necessary and sufficient conditions for a direct sum of modules with local endomorphism rings to be a CS-module. Since each indecomposable summand of a CS-module is uniform, it is enough to consider the direct sums of uniform modules with local endomorphism rings. The proof of our main theorem will be based on a number of preparatory lemmas.

LEMMA 3.1. Let $M=\oplus_{i \in I} M_{i}$ be a direct sum of uniform modules with local endomorphism rings such that every uniform submodule of $M$ is essential in a direct summand of $M$. Let $j$ be an index in $I, A$ a nonzero submodule of $M_{j}$ and $f$ : $A \rightarrow \oplus_{i \in I \backslash j} M_{i}$ a non-monomorphic homomorphism. Then $f$ can be extended to a homomorphism $f^{*}: M_{j} \rightarrow \oplus_{i \in \backslash \backslash j} M_{i}$.

PROOF. Define $A^{*}=\{a-f(a) \mid a \in A\}$. Then $A^{*}$ is a submodule of $M$ and clearly $A^{*} \cap\left(\oplus_{i \in \Lambda \backslash j} M_{i}\right)=0$. Also, since $A^{*} \approx A, A^{*}$ is uniform and so by hypothesis $A^{*}$ is essential in a direct summand $D$ of $M$. Then $D$ is also uniform and, by [1, Theorem 12.6], $D$ is isomorphic to some $M_{k}(k \in I)$. Hence $D$ has a local endomorphism ring, and it follows by Lemma 2.1 that $D$ has the exchange property. There are submodules $N_{i}$ of $M_{i}$ such that $D \oplus\left(\oplus_{i \in I} N_{i}\right)=M$. Then, for each $i, N_{i}$ is a direct summand of $M_{i}$, and since $M_{i}$ is uniform, we get $N_{i}=M_{i}$ or $N_{i}=0$. Hence it follows easily that there exists an index $t \in I$ such that $D \oplus\left(\oplus_{i \in I \backslash t} M_{i}\right)=\oplus_{i \in I} M_{i}$. Suppose that $t \neq j$. Then we have $M=D \oplus M_{j} \oplus\left(\oplus_{I \backslash t \cup j} M_{i}\right)$. So, in particular, we have $D \cap M_{j}=0$. This implies that $A^{*} \cap M_{j}=0$, and since $M_{j}$ is uniform, $A^{*} \cap A=0$. This means that $f(a) \neq 0$ for each nonzero $a \in A$, that is $f$ is a monomorphism, a contradiction. Thus we have $t=j$, hence $M=D \oplus\left(\oplus_{i \in I \backslash j} M_{i}\right)$. Let $p$ be the projection $D \oplus\left(\oplus_{i \in I \backslash j} M_{i}\right) \rightarrow \oplus_{i \in I \backslash j} M_{i}$, and we denote by $f^{*}$ the restriction of $p$ to $M_{j}$. Then clearly $f^{*}$ extends $f: A \rightarrow \oplus_{i \in I \backslash j} M_{i}$ which proves the lemma.

LEMMA 3.2. Let $R$ be any ring and $M=\oplus_{i \in I} M_{i}$ a direct sum of uniform right $R$-modules with local endomorphism rings. Assume further that every uniform submodule of $M$ is essential in a direct summand of $M$. Then for any choice of elements $x_{n} \in M_{i_{n}}$, with distinct $i_{n} \in I$, such that $\cap_{n=1}^{\infty} r_{R}\left(x_{n}\right) \supseteq r_{R}(y)$ for some $y \in M_{j}, j \in I$, the ascending sequence $\cap_{n=m}^{\infty} r_{R}\left(x_{n}\right), m=1,2,3, \ldots$ becomes stationary.

PROOF. Without loss of generality, we may assume for simplicity that $I=\{0,1,2$, $\ldots\}$ and $M=\oplus_{i=0}^{\infty} M_{i}$. We shall write $r(x)$ instead of $r_{R}(x)$, for $x \in M$. Choose any $x_{i} \in M_{i}, i=0,1,2, \ldots$, such that $r\left(x_{0}\right) \subseteq r\left(x_{i}\right)$ for all $i=1,2, \ldots$ We have to show that the sequence $K_{n}=\cap_{i=n}^{\infty} r\left(x_{i}\right), n=1,2, \ldots$, becomes stationary. 
Let $m$ be the first natural number such that $r\left(x_{0}\right) \neq K_{m}=\cap_{i=m}^{\infty} r\left(x_{i}\right)$. Then we have

$$
r\left(x_{0}\right)=\cap_{i=1}^{\infty} r\left(x_{i}\right)=\cdots=\cap_{i=m-1}^{\infty} r\left(x_{i}\right) \varsubsetneqq \cap_{i=m}^{\infty} r\left(x_{i}\right) \subseteq \cdots .
$$

Then, instead of $x_{0}, x_{1}, x_{2}, \ldots$, we shall consider the subsequence $x_{0}, x_{m}, x_{m+1}, \ldots$ Thus, without loss of generality, we may assume that $m=1$, that is $r\left(x_{0}\right) \neq n_{i=1}^{\infty} r\left(x_{i}\right)$.

We define a homomorphism

$$
\varphi: x_{0} R \longrightarrow \prod_{i=1}^{\infty} M_{i}
$$

by the following rule: for $t \in R, \varphi\left(x_{0} t\right)=\left(x_{1} t, x_{2} t, \ldots\right)$. If $x_{0} t=x_{0} t^{\prime}$, then $x_{i} t=x_{i} t^{\prime}$ for all $i \geq 1$, hence $\varphi$ is a well-defined homomorphism.

Let $I=\cup_{n=1}^{\infty} K_{n}=\cup_{n=1}^{\infty}\left(\cap_{i=n}^{\infty} r\left(x_{i}\right)\right)$; then $I$ is a right ideal of $R$ and $x_{0} I$ is a submodule of $x_{0} R$. Let $\bar{\varphi}$ be the restriction of $\varphi$ to $x_{0} I$. Take any $t \in I$; then $t \in K_{n}$ for some $n \geq 1$, hence $x_{i} t=0$ for each $i \geq n$. Thus

$$
\bar{\varphi}\left(x_{0} t\right)=\left(x_{1} t, \ldots, x_{n-1} t, 0,0,0, \ldots\right) \in \oplus_{i=1}^{\infty} M_{i},
$$

so $\bar{\varphi}$ is a homomorphism from $x_{0} I$ to $\oplus_{i=1}^{\infty} M_{i}$. Since $r\left(x_{0}\right) \neq \cap_{i=1}^{\infty} r\left(x_{i}\right)$ there exists a nonzero element $t^{\prime} \in R$ such that $t^{\prime} \in \cap_{i=1}^{\infty} r\left(x_{i}\right)$ but $t^{\prime} \notin r\left(x_{0}\right)$. It follows that $x_{0} t^{\prime} \neq 0$ but $\bar{\varphi}\left(t^{\prime}\right)=0$, hence $\bar{\varphi}$ is not a monomorphism. By Lemma 3.1, $\bar{\varphi}$ can be extended to a homomorphism $\psi: M_{0} \longrightarrow \oplus_{i=1}^{\infty} M_{i}$. Let $\bar{\psi}$ be the restriction of $\psi$ to $x_{0} R$, we have

$$
\varphi\left(x_{0}\right) I=\bar{\varphi}\left(x_{0} I\right)=\bar{\psi}\left(x_{0} I\right) \subseteq \bar{\psi}\left(x_{0} R\right)=\bar{\psi}\left(x_{0}\right) R \subseteq \oplus_{i=1}^{m} M_{i}
$$

for some $m \geq 1$. But $\varphi\left(x_{0}\right) I=\left(x_{1}, \ldots, x_{n}, \ldots\right) I$, so it follows that $x_{m+1} I=x_{m+2} I=$ $\cdots=0$. This means that $I \subseteq r\left(x_{m+1}\right) \cap r\left(x_{m+2}\right) \cap \cdots=K_{m+1}$. Therefore $I=K_{m+1}$ which completes the proof.

LEMMA 3.3. Let $M=\oplus_{i \in I} M_{i}$ be a direct sum of uniform modules with local endomorphism rings, and assume that every uniform submodule of $M$ is essential in a direct summand of $M$. Then for any infinite sequence of non-monomorphisms $f_{n}$

$$
M_{i_{1}} \stackrel{f_{1}}{\rightarrow} M_{i_{2}} \stackrel{f_{2}}{\rightarrow} \cdots \rightarrow M_{i_{n}} \stackrel{f_{n}}{\rightarrow} \cdots
$$

with distinct $i_{n} \in I$, and for any nonzero element $x_{1} \in M_{i_{1}}$, there exists $m \geq 1$ such that $f_{m} f_{m-1} \cdots f_{1}\left(x_{1}\right)=0$.

PROOF. Take any nonzero element $x_{1} \in M_{i_{1}}$ and put $x_{n}=f_{n-1} \cdots f_{1}\left(x_{1}\right)$, for $n \geq 2$. Then $x_{n} \in M_{i_{n}}$ and clearly $r\left(x_{1}\right) \subseteq r\left(x_{2}\right) \subseteq \cdots \subseteq r\left(x_{n}\right) \subseteq \cdots$. Since 
$r\left(x_{n}\right)=\cap_{i=n}^{\infty} r\left(x_{i}\right)$, it follows by Lemma 3.2 that this ascending sequence must become stationary. Thus there exists $m \geq 1$ such that $r\left(x_{m}\right)=r\left(x_{m+1}\right)$, that is $r\left(x_{m}\right)=$ $r\left(f_{m}\left(x_{m}\right)\right)$. This means that $x_{m} t=0$ for $t \in R$ if and only if $f_{m}\left(x_{m} t\right)=0$. Let $\bar{f}_{m}$ be the restriction of $f_{m}$ to $x_{m} R$; then $\bar{f}_{m}$ is a monomorphism. Suppose that $x_{m} R \neq 0$. Then because $M_{m}$ is uniform, it follows that $f_{m}$ is also a monomorphism, a contradiction to the hypothesis. Therefore we have $x_{m} R=0$, that is $x_{m}=f_{m-1} \cdots f_{1}\left(x_{1}\right)=0$.

We are now ready to give necessary and sufficient conditions for a direct sum of modules with local endomorphism rings to be a CS-module.

THEOREM 3.4. Let $R$ be any ring and $M=\oplus M_{i}$ a direct sum of uniform right $R$-modules $M_{i}$ with local endomorphism rings. Then the following conditions are equivalent:

(a) Every uniform submodule of $M$ is essential in a direct summand of $M$, and there does not exist an infinite sequence of non-isomorphic monomorphisms $f_{n}$ :

$$
M_{i_{1}} \stackrel{f_{1}}{\rightarrow} M_{i_{2}} \stackrel{f_{2}}{\rightarrow} \cdots \rightarrow M_{i_{n}} \stackrel{f_{n}}{\rightarrow} \cdots
$$

with all $i_{n}$ distinct in $I$;

(b) $M$ is a CS-module;

ProOF. (a) implies (b). Suppose that (a) holds. First we show that the family $\left\{M_{i} \mid i \in I\right\}$ is locally semi- $T$-nilpotent. Take any countable sequence $i_{1}, i_{2}, i_{3}, \ldots$ of pairwise different elements of $I$ and a sequence of non-isomorphisms $f_{n}$ :

$$
M_{i_{1}} \stackrel{f_{1}}{\rightarrow} M_{i_{2}} \stackrel{f_{2}}{\rightarrow} \cdots \rightarrow M_{i_{n}} \stackrel{f_{n}}{\rightarrow} \cdots
$$

By hypothesis, there exist infinitely many $f_{n}$ 's which are non-monomorphisms, say $f_{n(1)}, f_{n(2)}, \ldots, f_{n(k)}, \ldots$ with $n(1)<n(2)<\cdots<n(k)<\cdots$. Then we get an infinite sequence of non-monomorphisms $f_{n(k+1)-1} \cdots f_{n(k)}: M_{i_{n(k)}} \rightarrow M_{i_{n(k+1)}}$. Thus, by applying Lemma 3.3, we get easily that for any nonzero element $x \in M_{i_{1}}$, there exists $m \geq 1$ such that $f_{m} \cdots f_{1}\left(x_{1}\right)=0$. Therefore, the family $\left\{M_{i} \mid i \in I\right\}$ is locally semi- $T$-nilpotent. By Lemma 2.2, every local direct summand of $M$ is also a direct summand.

Now let $A$ be any nonzero complement submodule of $M$. Any cyclic submodule of $M$ has finite uniform dimension, so in particular $A$ contains a nonzero uniform submodule $U$. Let $U^{\prime}$ be a maximal essential extension of $U$ in $A$, then $U^{\prime}$ is uniform and a complement submodule of $A$. It follows by [3, Proposition 2.2] that $U^{\prime}$ is also a complement submodule of $M$. By Zorn's lemma, there exists a maximal local direct summand $\left\{V_{\alpha} \mid \alpha \in \Omega\right\}$ in $A$, where each $V_{\alpha}$ is an uniform summand of $M$. As we have shown above, $V=\oplus_{\alpha \in \Omega} V_{\alpha}$ is also a direct summand of $M$. Let $M=V \oplus M^{\prime}$ 
for some submodule $M^{\prime}$, then $A=V \oplus\left(M^{\prime} \cap A\right)$. If $M^{\prime} \cap A \neq 0$, then since $M^{\prime} \cap A$ is also a complement submodule of $M$ by [3, Proposition 2.2], $M^{\prime} \cap A$ contains a nonzero uniform summand of $M$ which contradicts the maximality of the local direct summand $V=\oplus_{\alpha \in \Omega} V_{\alpha}$. Hence $M^{\prime} \cap A=0$, that is $A=V$ is a direct summand of $M$. This proves that $M$ is a CS-module.

(b) implies (a). Suppose that $M$ is a CS-module. That every uniform submodule of $M$ is essential in a direct summand is obvious by the definition of CS-modules. The latter part of (a) was already proved in Baba-Harada [2, Proposition 3], by using the factor categories technique (cf. $[10,11]$ ). We include here a direct module-theoretic proof using an idea due to $R$. Wisbauer. Suppose that there exists an infinite sequence of non-isomorphic monomorphisms $f_{n}$ :

$$
M_{i_{1}} \stackrel{f_{1}}{\rightarrow} M_{i_{2}} \stackrel{f_{2}}{\rightarrow} \cdots \rightarrow M_{i_{n}} \stackrel{f_{n}}{\rightarrow} \cdots
$$

with distinct $i_{n}$. For simplicity we may assume that $I=\{1,2,3, \ldots\}$ and write $i_{n}=n$. Define $M_{n}^{*}=\left\{x_{n}-f_{n}\left(x_{n}\right) \mid x_{n} \in M_{n}\right\}$. Then it is easy to check that $\sum_{i=1}^{\infty} M_{i}^{*}$ is direct and $\left(\oplus_{i=1}^{n} M_{i}^{*}\right) \oplus M_{n+1}=\oplus_{i=1}^{n+1} M_{i}$ for each $n$. Hence $M^{*}=\oplus_{i=1}^{\infty} M_{i}^{*}$ is a local direct summand of $M$. Since $M$ is a CS-module, $M^{*}$ is essential in a direct summand $N$ of $M$ and we have $M=N \oplus L$. Assume that $L \neq 0$. By [1, Theorem 12.6 (1)], $L$ contains a nonzero indecomposable direct summand $X$. Let $L=Y \oplus X$, then $M=N \oplus Y \oplus X$. Since $N \oplus Y$ is a maximal direct summand in the sence of [1], by [1, Theorem 12.6 (2)] we have $M=N \oplus Y \oplus M_{k}$ for some $M_{k}$. This implies that $M_{k} \oplus M_{k}^{*}$ is a direct summand of $\mathrm{M}$. However, it is obvious that $M_{k} \oplus M_{k}^{*}$ is essential in $M_{k} \oplus M_{k+1}$. Thus $M_{k} \oplus M_{k}^{*}=M_{k} \oplus M_{k+1}$ implying that $f_{k}$ is epimorphic, hence an isomorphism, a contradiction. Therefore $L=0$, that is $M^{*}$ is essential in $M$. In particular, we have $M^{*} \cap M_{1} \neq 0$. Take any nonzero element $x_{1} \in M_{1} \cap M^{*}$. Then

$$
x_{1}=y_{1}-f_{1}\left(y_{1}\right)+y_{2}-f_{2}\left(y_{2}\right)+\cdots+y_{n}-f_{n}\left(y_{n}\right),
$$

where $y_{i} \in M_{i}, 1 \leq i \leq n$. It follows that $y_{1}=x_{1}, y_{i}=f_{i-1}\left(y_{i-1}\right)$ for $2 \leq i \leq n$ and $f_{n}\left(y_{n}\right)=0$. Hence $f_{n} f_{n-1} \cdots f_{1}\left(x_{1}\right)=0$ which is a contradiction because all $f_{1}, \ldots, f_{n}$ are monomorphisms.

COROLLARY 3.5. Let $R$ be any ring and $M=\oplus_{i \in I} M_{i}$ a direct sum of right $R$ modules $M_{i}$ with local endomorphism rings. Assume that $M$ is a CS-module. Then this decomposition complements direct summands and $M$ has the exchange property. Moreover, in this case $S=$ End $M$ is a semi-regular ring.

PROOF. By the proof of Theorem 3.4 ((a) implies (b)), we have that if the CS-module $M$ has the decomposition $M=\oplus_{i \in I} M_{i}$, where each $M_{i}$ has a local endomorphism ring, then the family $\left\{M_{i} \mid i \in I\right\}$ is locally semi-T-nilpotent. Now the result follows from Lemma 2.2 . 
The result below is an easy consequence of Lemma 3.2.

COROLlaRY 3.6. Let $M=\oplus_{i \in I} M_{i}$ be a direct sum of indecomposable quasiinjective modules $M_{i}$. Then the following conditions are equivalent:

(a) $M$ is quasi-injective.

(b) $M_{i}$ is $M_{j}$-injective for all $i \neq j$ in $I$, and every uniform submodule of $M$ is essential in a direct summand of $M$.

PROOF. (a) implies (b). This is immediate by the well-known facts that if $A$ and $B$ are modules with $A \oplus B$ quasi-injective, then $A$ is $B$-injective and $B$ is $A$-injective (see for example [14, Proposition 1.17]), and that each quasi-injective module is CS.

(b) implies (a). Suppose that (b) holds. Note that each $M_{i}$ is uniform with a local endomorphism ring. By Lemma 3.2, for any choice of elements $x_{n} \in M_{i_{n}}, n=$ $1,2, \ldots$, with distinct $i_{n} \in I$, such that $\cap_{n=1}^{\infty} r\left(x_{n}\right) \supseteq r(y)$ for some $y \in M_{j}, j \in I$, the ascending sequence $\cap_{n=m}^{\infty} r\left(x_{n}\right), m=1,2,3, \ldots$, becomes stationary. Now it follows by Lemma 2.3 that $M$ is quasi-injective.

The next corollary generalizes the Clark-Huynh result in [4] which was mentioned in the Introduction.

COROLLARY 3.7. Let $R$ be a semiperfect right self-injective ring. Then $R$ is $\mathrm{QF}$ if and only if every uniform submodule of the free right $R$-module $R^{(\mathbb{N})}$ is contained in a finitely generated submodule of $R^{(\mathbb{N})}$.

PROOF. If $R$ is QF then $R^{(\mathbb{N})}$ is injective, and so every uniform submodule $U$ of $R^{(\mathbb{N})}$ is contained in an injective envelope $A$ of $U$ in $R^{(\mathbb{N})}$. Since $A$ is indecomposable projective, $A$ must be cyclic by [1, Proposition 27.10]. Conversely, suppose that $R$ is semiperfect right self-injective, and consider the free right $R$-module $R^{(\mathbb{N})}$. Then $R^{(\mathbb{N})}$ can be decomposed as $R^{(\mathrm{N})}=\oplus_{i \in I} A_{i}$, where each $A_{i}$ is an indecomposable injective right $R$-module. Let $U$ be any uniform submodule of $R^{(\mathbb{N})}$, then by hypothesis, there is a finite subset $F \subset I$ such that $U \subseteq \oplus_{i \in F} A_{i}$. But $\oplus_{i \in F} A_{i}$ is injective, hence $U$ is essential in a direct summand of $\oplus_{i \in F} A_{i}$, thus of $R^{(\mathbb{N})}$. By Corollary 3.6, we have that $R^{(\mathbb{N})}$ is quasi-injective, so $R^{(\mathbb{N})}$ is $(R-)$ injective. Therefore $R$ is a QF-ring (see for example [1, Theorems 25.1 and 31.9]).

As a further application of Theorem 3.4, we next give a criterion of when a ring $R$ is right $\Sigma$-CS, that is any direct sum of copies of $R_{R}$ is a CS-module. Right nonsingular right $\Sigma$-CS rings are precisely the right nonsingular rings over which nonsingular right modules are projective (see Goodearl [9, Chapter 5]). Right $\Sigma$-CS-rings in the general case were studied extensively by Oshiro (for example [15]) under the name 
right co- $H$-rings (in honor of Harada). Fore more recent results on $\Sigma$-CS rings and modules we refer to Clark and Wisbauer [5], Dung and Smith [7].

A module $M$ is called continuous if $M$ is a CS-module and every submodule of $M$ which is isomorphic to a direct summand of $M$ is also a direct summand of $M$ (see [14]).

COROLLARY 3.8. Let $R$ be a ring with a finite set of orthogonal primitive idempotents $e_{1}, \ldots, e_{n}$. Then the following conditions are equivalent:

(a) Each $e_{i} R \quad(1 \leq i \leq n)$ is a continuous right $R$-module, and every uniform submodule of $R_{R}^{(\mathbb{N})}$ is essential in a direct summand of $R_{R}^{(\mathbb{N})}$;

(b) $R$ is right $\Sigma$-CS;

ProOF. (a) implies (b). Assume (a). Note that each $e_{i} R$ is uniform and has a local endomorphism ring (see [14, Proposition 3.5]). Consider the free module $R_{R}^{(\mathbb{N})}$ which can be decomposed as $R_{R}^{(\mathbb{N})}=\oplus_{j \in J} M_{j}$, where each $M_{j}$ is isomorphic to some $e_{i} R,(1 \leq i \leq n)$. Suppose that there exists an infinite sequence of non-isomorphic monomorphisms $f_{n}$

$$
M_{j_{1}} \stackrel{f_{1}}{\rightarrow} M_{j_{2}} \stackrel{f_{2}}{\rightarrow} \cdots \rightarrow M_{j_{n}} \stackrel{f_{n}}{\rightarrow} \cdots
$$

Since among $\left\{M_{j} \mid j \in J\right\}$ there is only a finite number of non-isomorphic members, there are $k$ and $\ell$ with $k<\ell$ such that $M_{j_{k}} \approx M_{j_{\ell}}$. Since $M_{j_{k}}$ is indecomposable continuous, $f_{\ell-1} \ldots f_{k}$ is a monomorphism $M_{j_{k}} \rightarrow M_{j_{\ell}}$, it follows that $f_{\ell-1} \ldots f_{k}$ is an isomorphism, and this clearly implies that all $f_{\ell-1}, \ldots, f_{k}$ are isomorphisms, a contradiction. Thus there does not exist an infinite sequence of non-isomorphic monomorphisms

$$
M_{j_{1}} \stackrel{f_{1}}{\rightarrow} M_{j_{2}} \stackrel{f_{2}}{\rightarrow} \cdots \rightarrow M_{j_{n}} \stackrel{f_{n}}{\rightarrow} \cdots
$$

By Theorem 3.4, we have that $R_{R}^{(\mathbb{N})}$ is a CS-module. Now the result follows from the fact that if the ring $R$ is semiperfect and $R_{R}^{(\mathbb{N})}$ is CS, then $R$ is right $\Sigma$-CS (see Clark and Wisbauer [5] or Vanaja [18]).

(b) implies (a). This follows from Oshiro [15, Lemma 3.7] (cf. Clark and Wisbauer [5, Theorem 2.6] for an alternative proof).

REMARK. The proof of Corollary 3.8 yields also Theorem 1 ((a) implies (c)) in Clark and Huynh [4] which states that a ring $R$ is QF if (and only if) $R$ is semiperfect right continuous such that $R_{R}^{(n)}$ is CS for each $n \geq 1$ and every uniform submodule of any projective right $R$-module is contained in a finitely generated submodule. In fact, under these hypotheses, the above proof shows that every free right $R$-module is CS, that is $R$ is right $\Sigma$-CS. Since $R$ is right continuous, we have $J(R)=Z\left(R_{R}\right)$, 
where $J(R)$ and $Z\left(R_{R}\right)$ are the Jacobson radical and the singular right ideal of $R$, respectively. By Oshiro [15, Theorem 4.3], we deduce that $R$ is QF.

In view of Corollary 3.8, below we present an example of a semiperfect Noetherian ring $R$ such that every uniform submodule of any projective right $R$-module $P$ is essential in a direct summand of $P$, but $R$ is not semiprimary, so in particular $R$ is not right $\Sigma$-CS (see [15, Theorem 3.18]).

EXAMPLE 3.9. Let $R$ be a (left and right) hereditary serial Noetherian semiprime ring with zero left and right socles. For the existence of such rings $R$ we refer to Warfield [20, Theorems 5.11 and 5.14]. Clearly $R$ is semiperfect, but not semiprimary. By [20, Theorem 4.6], every finitely generated nonsingular right $R$-module is projective. Since $R$ is right nonsingular, this means that every finitely generated projective right $R$-module is a CS-module. Now let $P$ be any projective right $R$-module and $A$ a uniform submodule of $P$. By [1, Theorem 27.11], we have $P=\oplus_{i \in I} P_{i}$, where each $P_{i}$ is isomorphic to an indecomposable direct summand of $R_{R}$. It is easy to see that $A$ can be imbedded into a finite direct sum $P_{i_{1}} \oplus \cdots \oplus P_{i_{n}}$. Since each $P_{i_{k}}$ is Noetherian, it follows that $A$ is Noetherian, in particular $A$ is finitely generated. Thus $A$ is contained in a finite direct sum $P_{j_{1}} \oplus \cdots \oplus P_{j_{m}}$ which is a CS-module. Therefore $A$ is essential in a direct summand of $P_{j_{1}} \oplus \cdots \oplus P_{j_{m}}$, and hence of $P$.

We conclude the paper with a result which shows that, under certain chain conditions on the ring $R$, there is a rather large class of CS right $R$-modules which have decompositions into indecomposable summands with local endomorphism rings. The proof is inspired by some ideas in Clark and Wisbauer [5]. Recall that a module $M$ is said to be $\Sigma$-quasi-injective if any direct sum of copies of $M$ is also quasi-injective, and it is well known that $M$ is $\Sigma$-quasi-injective if and only if $M^{(\mathbb{N})}$ is quasi-injective.

THEOREM 3.10. Let $R$ be a ring and $M$ a finitely generated right $R$-module. Suppose that $M^{(\mathbb{N})}$ is a CS-module and $R$ satisfies ACC on $r_{R}(X)$ for finite subsets $X$ of $M$. Then $M$ is a finite direct sum of indecomposable $\Sigma$-quasi-injective submodules.

PROOF. Since $M$ can be decomposed as a finite direct sum of uniform submodules (see for example [14, Theorem 2.17 and Proposition 2.18]), we may assume, without loss of generality, that $M$ is uniform, and we have to show that $M$ is $\Sigma$-quasi-injective.

Since $R$ satisfies ACC on $r_{R}(X)$ for finite subsets $X$ of $M$, it follows that $R$ has ACC on $r_{R}(a)$ for elements $a \in M^{(\mathbb{N})}$. Since $M^{(\mathbb{N})}$ is CS, by [14, Proposition 2.18] we have that every local direct summand of $M^{(\mathbb{N})}$ is also a direct summand. Next we show that $S=$ End $M$ is local. Let $a_{1}, a_{2}, \ldots, a_{n}, \ldots$ be any sequence of elements in $S$ and consider the descending chain of principal left ideals of $S$

$$
S a_{1} \supset S a_{2} a_{1} \supset \cdots \supset S a_{n} a_{n-1} \cdots a_{1} \supset \cdots .
$$


Let $M^{(\mathbb{N})} \approx \oplus_{n=1}^{\infty} M_{n}$ with $M_{n} \approx M$ for each $n \geq 1$. We may consider $a_{n}$ as a homomorphism from $M_{n}$ to $M_{n+1}$. Define $\dot{M}_{n}^{*}=\left\{x_{n}-a_{n}\left(x_{n}\right) \mid x_{n} \in M_{n}\right\}$. As in the proof of Theorem 3.4 ((b) implies (a)), we have that $\oplus_{n=1}^{\infty} M_{n}^{*}$ is a local direct summand of $M^{(\mathbb{N})}$, hence $\oplus_{n=1}^{\infty} M_{n}^{*}$ is a direct summand of $M^{(\mathbb{N})}$. Since $M$ is finitely generated, by Wisbauer [21, 43.3], there exist a positive integer $m$ and an element $h \in S$ such that $a_{m-1} \cdots a_{1}=h a_{m} a_{m-1} \cdots a_{1}$. This implies that $S a_{m-1} \cdots a_{1}=S a_{m} a_{m-1} \cdots a_{1}$. It follows that $S$ satisfies $D C C$ on principal left ideals, that is $S$ is a right perfect ring. But $M$ is indecomposable, hence $S$ contains no nontrivial idempotents, so $S$ is a local ring.

By hypothesis, $M$ is a CS-module, so by Theorem 3.4 ((b) implies (a)), every monomorphism $g: M \rightarrow M$ is also an isomorphism. Since End $M$ is local and $M \oplus M$ is CS, the above fact and [8, Lemma 3] show that $M$ is $M$-injective, that is $M$ is quasi-injective. By Corollary 3.6, we have that $M^{(\mathbb{N})}$ is quasi-injective, hence $M$ is $\Sigma$-quasi-injective which completes the proof.

\section{Acknowledgement}

This work was supported by the National Basic Research Programme in Natural Sciences of Vietnam. The author would like to thank the referee for some helpful comments.

\section{References}

[1] F. W. Anderson and K. R. Fuller, Rings and categories of modules, second edition, Graduate Texts in Math. 13 (Springer, Berlin, 1992).

[2] Y. Baba and M. Harada, 'On almost $M$-projectives and almost $M$-injectives', Tsukuba J. Math. 14 (1990), 53-69.

[3] A. W. Chatters and C. R. Hajarnavis, 'Rings in which every complement right ideal is a direct summand', Quart. J. Math. Oxford Ser. (2) 28 (1977), 61-80.

[4] J. Clark and Dinh Van Huynh, 'When is a self-injective semiperfect ring quasi-Frobenius', $J$. Algebra 165 (1994), 531-542.

[5] J. Clark and R. Wisbauer, ' $\Sigma$-extending modules', J. Pure Appl. Algebra 104 (1995), 19-32.

[6] P. Crawley and B. Jónsson, 'Refinements for infinite direct decompositions of algebraic systems', Pacific J. Math. 14 (1964), 797-855.

[7] Nguyen V. Dung and P. F. Smith, ' $\Sigma$-CS-modules', Comm. Algebra 22 (1994), 83-93.

[8] , 'Rings for which certain modules are CS', J. Pure Appl. Algebra 102 (1995), 273-287.

[9] K. R. Goodearl, Ring theory (Marcel Dekker, New York, 1976).

[10] M. Harada, 'On categories of indecomposable modules II', Osaka J. Math. 8 (1971), 309-321.

[11] - Factor categories with applications to direct decomposition of modules, Lecture Notes in Pure and Applied Math. 88 (Marcel Dekker, New York, 1983).

[12] M. A. Kamal and B. J. Müller, 'The structure of extending modules over Noetherian rings', Osaka J. Math. 25 (1988), 539-551. 
[13] T. Kato, 'Self-injective rings', Tôhoku Math. J. 19 (1967), 485-495.

[14] S. H. Mohamed and B. J. Müller, Continuous and discrete modules, London Math. Soc. Lecture Note Series 147 (Cambridge University Press, Cambridge, 1990).

[15] K. Oshiro, 'Lifting modules, extending modules and their applications to $Q F$-rings', Hokkaido Math. J. 13 (1984), 310-338.

[16] B. L. Osofsky, 'A generalization of quasi-Frobenius rings', J. Algebra 4 (1966), 373-387.

[17] B. L. Osofsky and P. F. Smith, 'Cyclic modules whose quotients have all complement submodules direct summands', J. Algebra 139 (1991), 342-354.

[18] N. Vanaja, 'Characterization of rings using extending and lifting modules', in: Ring theory Proceedings of the Denison conference 1992 (eds. S. K. Jain and S. T. Rizvi) (World Scientific, Singapore, 1993) pp. 329-342.

[19] R. B. Warfield Jr., 'A Krull-Schmidt theorem for infinite sums of modules', Proc. Amer. Math. Soc. $22(1969), 460-465$.

[20] _ _ ' 'Serial rings and finitely presented modules', J. Algebra 37 (1975), 187-222.

[21] R. Wisbauer, Foundations of module and ring theory (Gordon and Breach, Reading, 1991).

[22] B. Zimmermann-Huisgen and W. Zimmermann, 'Classes of modules with the exchange property', J. Algebra 88 (1984), 416-434.

Institute of Mathematics

P.O. Box 631 Bo Ho

Hanoi

Vietnam 